\title{
Radial extracorporeal shock wave therapy promotes osteochondral regeneration of knee joints in rabbits
}

\author{
HUI QI ${ }^{1,2}$, SHAOFENG JIN ${ }^{1,2}$, CHUNYANG YIN ${ }^{3,4}$, LEI CHEN ${ }^{1,2}$, LEI SUN ${ }^{1,2}$ and YAJUN LIU ${ }^{5}$ \\ ${ }^{1}$ Musculoskeletal Tissue Bank, Beijing Jishuitan Hospital; ${ }^{2}$ Laboratory of Musculoskeletal Regenerative Medicine, \\ Beijing Institute of Traumatology and Orthopaedics, Beijing 100035; ${ }^{3}$ State Key Laboratory of Environmental Chemistry \\ and Ecotoxicology, Research Center for Eco-Environmental Sciences, Chinese Academy of Sciences, Beijing 100085; \\ ${ }^{4}$ University of Chinese Academy of Sciences, Beijing 100049; ${ }^{5}$ Orthopedic Shock Wave Treatment Center, \\ Spine Surgery Department, Beijing Jishuitan Hospital, Beijing 100035, P.R. China
}

Received December 29, 2017; Accepted July 6, 2018

DOI: $10.3892 / \mathrm{etm} .2018 .6631$

\begin{abstract}
Radial extracorporeal shock wave therapy (rESWT) has been proven to be effective for nonunion fractures. It was, thus, hypothesized that it may be used as a supplement therapy to promote osteochondral regeneration when combined with a scaffold previously prepared by our research group. In the present study, to verify this hypothesis, New Zealand white adult rabbits were anaesthetized and divided into three groups, as follows: Untreated control group, in which full-thickness cylindrical osteochondral defects were created without repairing; scaffold group, in which rabbits were implanted with the scaffolds; scaffold plus rESWT group, in which rabbits were implanted with scaffolds and then treated with rESWT at 2 weeks post-surgery. At 6 and 12 weeks after surgery, the animals were sacrificed. Nitric oxide (NO) levels in the synovial cavity of the knee joints were measured by the Griess method. In addition, macroscopic observation and the gross score according to the International Cartilage Repair Society (ICRS) histological scoring system were determined. Histological evaluation was also performed by hematoxylin-eosin and Safranin O/fast green staining. The results demonstrated that both the scaffold and scaffold plus rESWT treatments significantly reduced NO levels in the synovial cavity at 6 weeks after surgery $(\mathrm{P}<0.05)$, whereas no significant difference was observed at 12 weeks after surgery. The ICRS scores of the scaffold and scaffold plus rESWT groups were significantly higher in comparison with those in the control group $(\mathrm{P}<0.05)$, and rESWT further increased these scores at 12 weeks after surgery $(\mathrm{P}<0.05)$. Histological results
\end{abstract}

Correspondence to: Professor Yajun Liu, Orthopedic Shock Wave Treatment Center, Spine Surgery Department, Beijing Jishuitan Hospital, 31 Xinjiekou East Road, Xicheng, Beijing 100035, P.R. China

E-mail: drliuyajun@163.com

Key words: osteochondral defects, radial extracorporeal shock wave therapy, nitric oxide revealed that osteochondral regeneration was improved after treatment with scaffold or scaffold plus rESWT, with the latter displaying better results. These data suggested that rESWT improved the osteochondral regeneration when applied in combination with the scaffold, and that one of the underlying mechanisms may involve the reduction of $\mathrm{NO}$ in the synovial fluid. Therefore, rESWT may be a useful treatment for knee osteochondral regeneration.

\section{Introduction}

Articular cartilage tissue lacks blood supply and has a limited regeneration capacity. An osteochondral defect occurs when the subchondral bone is damaged due to abnormal mechanical loading (1-3). This type of defect is relatively common and notably reported in $\sim 20 \%$ of all arthroscopic procedures (4), and if left untreated or treated incorrectly, osteoarthritis (OA) will occur. Patients develop pain, locking of the joint and/or other symptoms, which may lead to disability. Various methods have been reported for the treatment of osteochondral defects, including the marrow stimulation technique, osteochondral autograft/allograft transplantation and autologous chondrocyte implantation (1-3). Although the majority of methods have been proven to improve the symptoms for a certain period of time, long-term efficacy and physiological property recovery needs to be further examined (4-6). Our research group has previously fabricated and implanted three-dimensional porous scaffolds into osteochondral defects in rabbits (7-10). Although repair was observed, it was difficult for a single scaffold to meet the needs for reconstructing the cartilage and subchondral bone at the same time. Improved regenerative effects are still under evaluation.

Two types of extracorporeal shock wave therapy (ESWT) have been used in the musculoskeletal system, including focused ESWT (fESWT) and radial ESWT (rESWT) (11). A larger number of studies have been conducted on fESWT in the clinical setting and animal models, as opposed to rESWT (12). As wave patterns and tissue penetrating depth are different for fESWT and rESWT, corresponding effects are expected to vary (12) and further investigations are required. It has previously been reported that rESWT on tendinopathy can be 
more effective compared with other types of treatment, such as traditional physiotherapy and surgery (13), while its role in osteochondral defects remains incompletely understood. Therefore, it can be hypothesized that improved repair may be achieved when combining scaffold transplantation with rESWT.

In the present study, a rabbit osteochondral defect model was used to assess the morphology of articular cartilage and subchondral bone according to the International Cartilage Repair Society (ICRS) histological scoring system. Nitric oxide (NO) levels in the synovial cavity were measured, and histological sections were examined by hematoxylin-eosin (H\&E) and Safranin O/fast green staining. By comparing these parameters among the untreated control, scaffold and scaffold plus rESWT groups, it was concluded that rESWT combined with the scaffold improved the osteochondral regeneration, which may be a result of the reduction of $\mathrm{NO}$ levels in the synovial fluid.

\section{Materials and methods}

Animal model. The experimental protocol was reviewed and approved by the Institutional Review Board of Beijing Jishuitan Hospital (Beijing, China). A total of 15 male New Zealand White rabbits aged 5 months (mean weight, $2.7 \mathrm{~kg}$; weight range, $2.5-2.9 \mathrm{~kg}$ ) were obtained from Xinglong Experimental Animal Farm (Beijing, China). Animals were kept at $16-28^{\circ} \mathrm{C}$ with $40-70 \%$ humidity under a 12-h light/dark cycle with free access to food and water. Acclimatization for 7 days was allowed prior to experiments. Under anesthesia performed by injection of $0.1 \%$ pentobarbital sodium $(40 \mathrm{mg} / \mathrm{kg})$, a model of osteochondral defects was established. During the surgery, a hole was drilled with a $3.5-\mathrm{mm}$ diameter (the diameter of the drill) and 2.21 $\pm 0.24-\mathrm{mm}$ depth in the weight-bearing osteochondral surface of the femoral condyle (Fig. 1). Surgery was performed in the bilateral knee joints of the rabbits; left knee joints were treated 12 weeks and right knee joints were treated for the last 6 weeks only. Rabbits $(n=5)$ were assigned the following groups: Control group with osteochondral defects not receiving treatment; scaffold group with osteochondral defects treated with a porous scaffold implant, which was prepared by the authors from dermal tissues of a calf back following decellularization, reconstruction, cross-linking and surface-modification as described previously (7-10); and scaffold + rESWT group with osteochondral defects treated with a scaffold implant and rESWT.

Shock wave treatment. Radial shock waves were applied using a STORZ device (STORZ Medical, Tägerwilen, Switzerland). At 2 weeks after surgery, rabbits in the scaffold plus rESWT group were anesthetized and then placed in a lateral position. A coupling gel was smeared to the skin surrounding the surgical area. The knee joint of each rabbit was subjected to 500 shock wave impulses at 1.5 bar every time (Fig. 2). Immediately after rESWT application, the knee joint was examined for swelling, ecchymosis or hematoma. All animals were sacrificed 12 weeks following the initial surgery.

NO quantification. Prior to euthanasia, the rabbits were anesthetized and the synovial cavity of knee joints was washed with $0.5 \mathrm{ml}$ sterile saline. The synovial exudates were collected by aspiration and stored at $-80^{\circ} \mathrm{C}$ prior to the assay. During the measurement, all samples were centrifuged at 1,000 x $\mathrm{g}$ for $20 \mathrm{~min}$ at $4^{\circ} \mathrm{C}$. Concentrations of $\mathrm{NO}_{\mathrm{X}}$ (nitrite/nitrate) in the synovial fluid were determined using a method based on the enzymatic conversion of nitrate to nitrite by nitrate reductase, followed by colorimetric detection of nitrite as an azo dye product of the Griess reaction (Molecular Probes; Thermo Fisher Scientific, Inc., Waltham, MA, USA). Briefly, $130 \mu \mathrm{l}$ deionized water, $150 \mu \mathrm{l}$ of the sample and $20 \mu \mathrm{l}$ Griess reagent were respectively added into each well of a 96 -well plate, and incubated for $30 \mathrm{~min}$ at room temperature. The absorbance of each sample was then measured spectrophotometrically at $540 \mathrm{~nm}$. The concentration of $\mathrm{NO}_{\mathrm{X}}$ was determined through the standard curve of nitrite concentration $(1-100 \mu \mathrm{M})$ against the absorbance at $540 \mathrm{~nm}$.

Macroscopic observation. The femoral condyles of rabbits in each group were observed without microscopic aid and images were captured with a Nikon D850 camera (Nikon Corporation, Tokyo, Japan). Macroscopic evaluation was conducted according to the ICRS scoring system (14). Briefly, the degree of defect repair, integration to border zone and macroscopic appearance were scored on a scale of $0-4$; normal tissue achieves a score of 12 .

Histological analyses. The femoral condyles were dissected into small sections, and the regenerated/defect part was fixed in 4\% paraformaldehyde for 3-4 days. Subsequently, the tissues were decalcified in 10\% EDTA solution (Sigma-Aldrich; Merck KGaA, Darmstadt, Germany) for 28 days and sectioned into $4-\mu \mathrm{m}$ slices. Sections were stained with conventional hematoxylin and eosin (H\&E). Sections were incubated with hematoxylin for $8-10 \mathrm{~min}$ and then $0.5 \%$ eosin for $20 \mathrm{sec}$, both at room temperature. Safranin O/fast green staining kit (Beijing Solarbio Science \& Technology Co., Ltd.) was used to determine the extent of cartilage repair and observe the cartilaginous specific matrix secretion according to the manufacturer's instructions. All the procedures of Safranin $\mathrm{O} /$ fast green staining were performed at room temperature. Following dewaxing with xylene $(3 \times 10 \mathrm{~min})$, sections were stained with Weigert solution for 3-5 min and washed with distilled water (2x1 min). After differentiation for $15 \mathrm{sec}$ with acid differentiation solution, sections were incubated with fast green for $5 \mathrm{~min}$ and then into safranin $\mathrm{O}$ for $2 \mathrm{~min}$. Each step was performed with $1 \mathrm{ml}$ solutions followed by washing with distilled water $(2 \times 1 \mathrm{~min})$. After staining with fast green and safranin $\mathrm{O}$, sections were washed with a weak acid solution for 1-2 min to remove the residual stain, and then also washed with distilled water 2 times for $1 \mathrm{~min}$. All the solutions were provided in the kit. Sections were then incubated into $95 \%$ alcohol and absolute ethanol for dehydration, each step was performed twice for a few seconds. Xylene was used for transparency prior to sealing the sections. Sections were analyzed using a light microscope (magnification, $\mathrm{x} 40$ ). The normal cartilage matrix is positively stained by Safranin $\mathrm{O}$ (red), while the injury cartilage that is negatively stained is almost white or weakly red.

Statistical analyses. Data are expressed as the mean \pm standard derivation. One-way analysis of variance followed by post hoc 

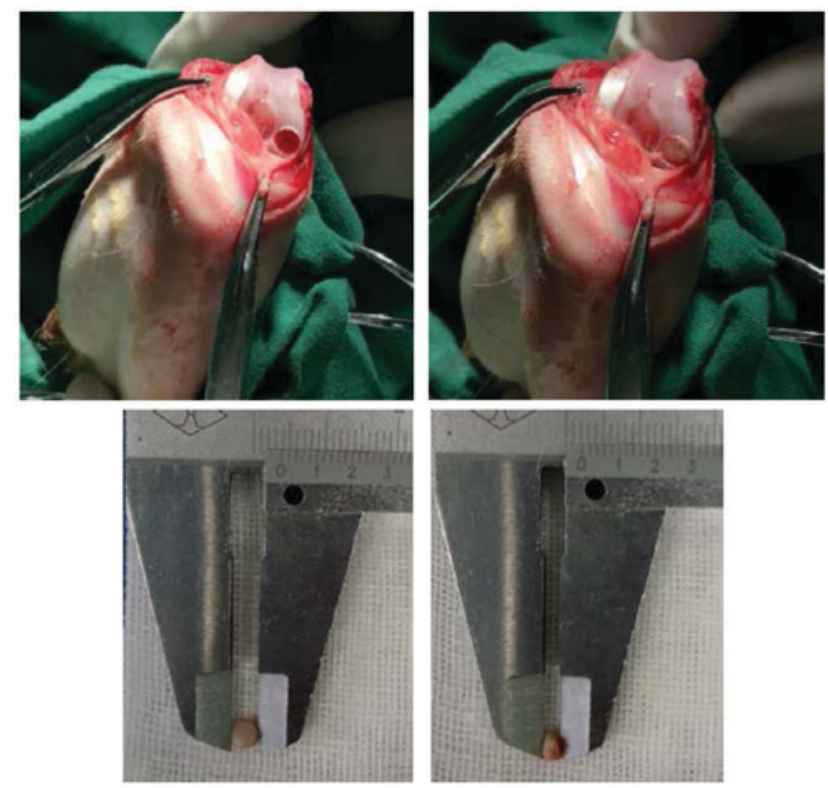

Figure 1. Osteochondral defect in the femoral condyle of a rabbit (top left) and the porous scaffold placed into the defect (top right) with suitable diameter (bottom left) and thickness (bottom right).

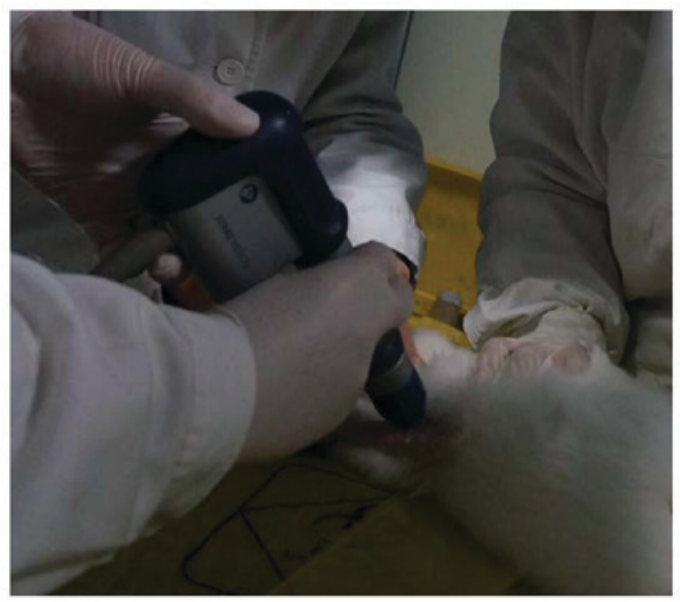

Figure 2. Radial extracorporeal shock wave therapy on osteochondral defect of the rabbit.

testing with Fisher's least significant difference was used to analyze experimental data with the IBM SPSS software (version 21.0; IBM Corp., Armonk, NY, USA). In the current study, statistically significant differences were indicated by $\mathrm{P}<0.05$.

\section{Results}

rESWT leads to lower $N O_{X}$ concentrations in the synovial fluid. The total concentration of $\mathrm{NO}_{\mathrm{X}}$, including nitrite and nitrate, in the synovial fluid was measured with the Griess method. $\mathrm{NO}_{\mathrm{X}}$ concentrations were significantly lower in the scaffold $(19.2 \pm 3.0 \mu \mathrm{mol} / \mathrm{l})$ and scaffold plus rESWT groups $(18.8 \pm 4.0 \mu \mathrm{mol} / \mathrm{l})$ as compared with the control group $(25.8 \pm 3.1 \mu \mathrm{mol} / 1)$ at 6 weeks after surgery. However, no significant difference was detected among the three groups at 12 weeks after surgery (Table I).
Table I. NO concentration in the synovial fluid $(n=5)$.

\begin{tabular}{llr}
\hline & \multicolumn{2}{c}{$\begin{array}{c}\text { NO concentration } \\
\text { post-surgery }(\mu \mathrm{mol} / \mathrm{l})\end{array}$} \\
\cline { 2 - 3 } Group & 6 weeks & 12 weeks \\
\hline Control & $25.8 \pm 3.1$ & $10.2 \pm 2.9$ \\
Scaffold & $19.2 \pm 3.0^{\mathrm{a}}$ & $8.0 \pm 1.4$ \\
Scaffold plus rESWT & $18.8 \pm 4.0^{\mathrm{a}}$ & $8.4 \pm 2.4$
\end{tabular}

${ }^{\text {aP }}<0.05$ vs. the control group. Data represent the mean \pm standard deviation. NO, nitric oxide; rESWT, radial extracorporeal shock wave therapy.

rESWT improves macroscopic osteochondral appearance. In order to identify whether rESWT helps promote osteochondral regeneration, the gross appearance of the three groups at 6 or 12 weeks after surgery was compared. Macroscopically, in the control group, the surface of the defect area was evidently lower in comparison with the normal tissue at 6 weeks after surgery. However, the scaffold and the scaffold plus rESWT groups exhibited marked improvement in osteochondral appearance after 6 and 12 weeks (Fig. 3A-F). The ICRS scores also revealed similar results $(\mathrm{P}<0.05$; Fig. 4). Additionally, the scaffold plus rESWT group displayed much better repair characteristics after 12 weeks, but not after 6 weeks of surgery (Figs. 3E and F, and 4). All these results indicated a significant therapeutic effect of rESWT used in combination with the scaffold.

rESWT results in improved osteochondral regeneration. To determine the effect of rESWT on the osteochondral defect of knee joints, H\&E staining was performed. As presented in Fig. 5, the defect area was covered by fibrous tissue both at week 6 and week 12 in the control group (Fig. 5A and B) and limited repair was observed in the scaffold group at 6 weeks following surgery when implanted with porous scaffold (Fig. 5C). A limited number of small rounded chondrocyte-type cells were detected beneath the defect area and at the interface with the normal tissue at 12 weeks following surgery (Fig. 5D). An increased number of rounded chondrocyte-type cells were detected and an increased cartilaginous-type surface area was presented by rESWT treatment group both at week 6 and week 12 (Fig. 5E and F). The results were further confirmed by Safranin O/fast green staining (Fig. 6). Safranin O/fast green staining was used to access the cartilage matrix content. The results exhibited that Safranin O staining was negative in the control group both at week 6 and week 12 (Fig. 6A and B) and weak-positive in the scaffold group, beneath the defect area and at the interface with the normal tissue both at week 6 and week 12 (Fig. 6C and D) and a limited number of small rounded chondrocyte-type cells were detected at 12 weeks following surgery (Fig. 6D). In the scaffold plus rESWT group, an increased amount of chondrocyte-type cells were observed. Safranin O staining was positive at week 6 (Fig. 6E) and a larger proportion of cartilaginous-matrix tissue was positively stained with Safranin O at week 12 (Fig. 6F). 
A

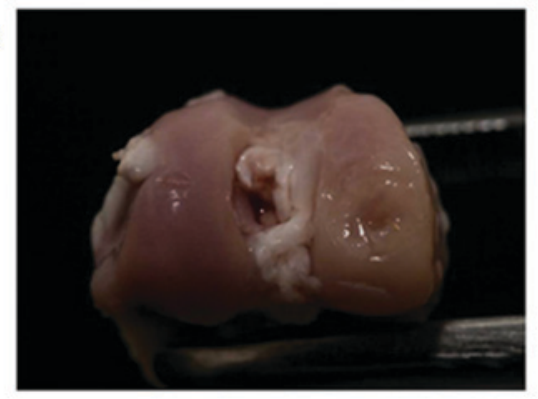

C

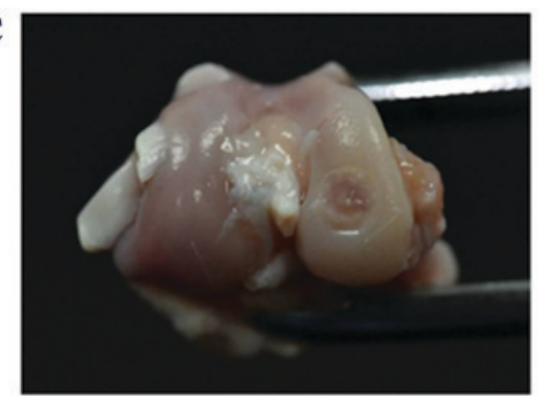

E

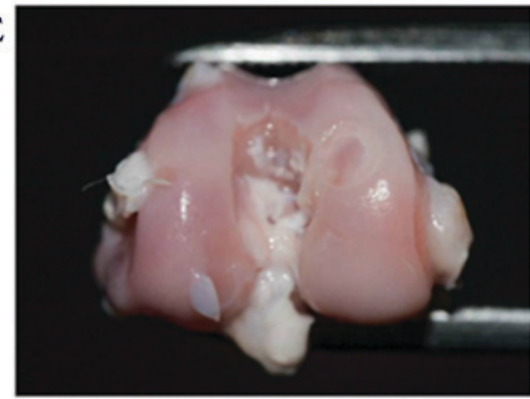

B

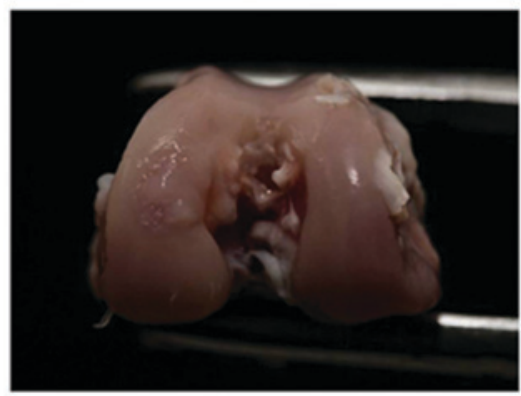

D

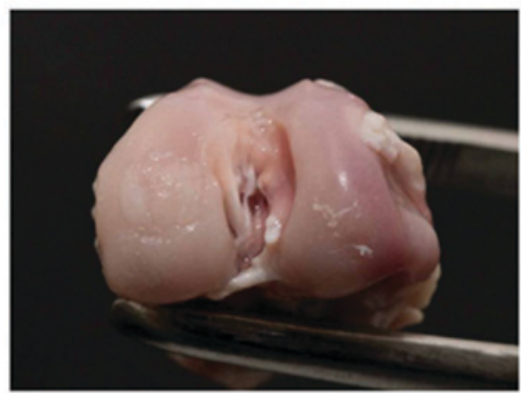

F

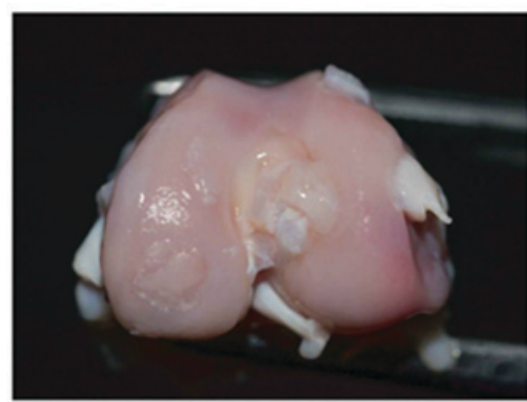

Figure 3. Macroscopic observation subsequent to surgery. Representative images of the untreated control at (A) 6 weeks and (B) 12 weeks after surgery. Representative images of the group implanted with a scaffold at (C) 6 weeks and (D) 12 weeks after surgery. Representative images of the group implanted with a scaffold and then treated with radial extracorporeal shock wave therapy, at (E) 6 weeks and (F) 12 weeks after surgery.

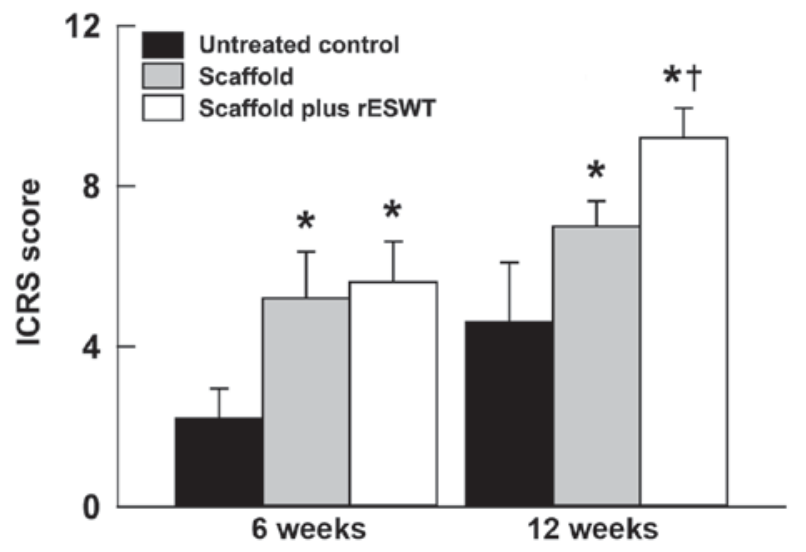

Figure 4. ICRS scores at 6 and 12 weeks after surgery. Data are presented as the mean \pm standard deviation. ${ }^{*} \mathrm{P}<0.05$ vs. untreated control group; ${ }^{\dagger} \mathrm{P}<0.05$ vs. scaffold group. ICRS, International Cartilage Repair Society; rESWT, radial extracorporeal shock wave therapy.

\section{Discussion}

Severe cartilage defects are often combined with the destruction of subchondral bone and are known as osteochondral defects. If left untreated, an osteochondral defect will gradually expand to the surrounding normal cartilage, causing OA (15).
While a number of treatment options currently present good short-term functional restoration, the majority of the treatments result in fibrocartilage with inferior mechanical properties and diminished long-term durability $(1,16)$. Therefore, to further improve the osteochondral regeneration, a combination of different methods is actively investigated currently (17).

A scaffold is one of the three main components of tissue engineering, along with cells and growth factors $(18,19)$. A porous scaffold was fabricated by our group and received a Chinese Invention Patent (patent no. ZL 20111 0099363.1) (7-10), was prepared from the dermal tissues of a calf back following decellularization, reconstruction, cross-linking and surface-modification. Although this scaffold promotes osteochondral regeneration, its effect was not fully satisfactory in terms of functional recovery. Previously, ESWT has been reported to serve an important role in treating a number of orthopedic injuries $(20,21)$. The Food and Drug Administration of USA has approved ESWT for the treatment of proximal plantar fasciitis, and clinical trials of lateral epicondylitis of the elbow, calcific tendinitis of the shoulder and nonunion fractures $(20,21)$. To date, an increasing number of studies have demonstrated the significant effect of ESWT on bone and tendon regeneration in animals and patients (13). There are also several studies reporting the therapeutic effect of ESWT on OA, osteochondritis dissecans or cartilage 

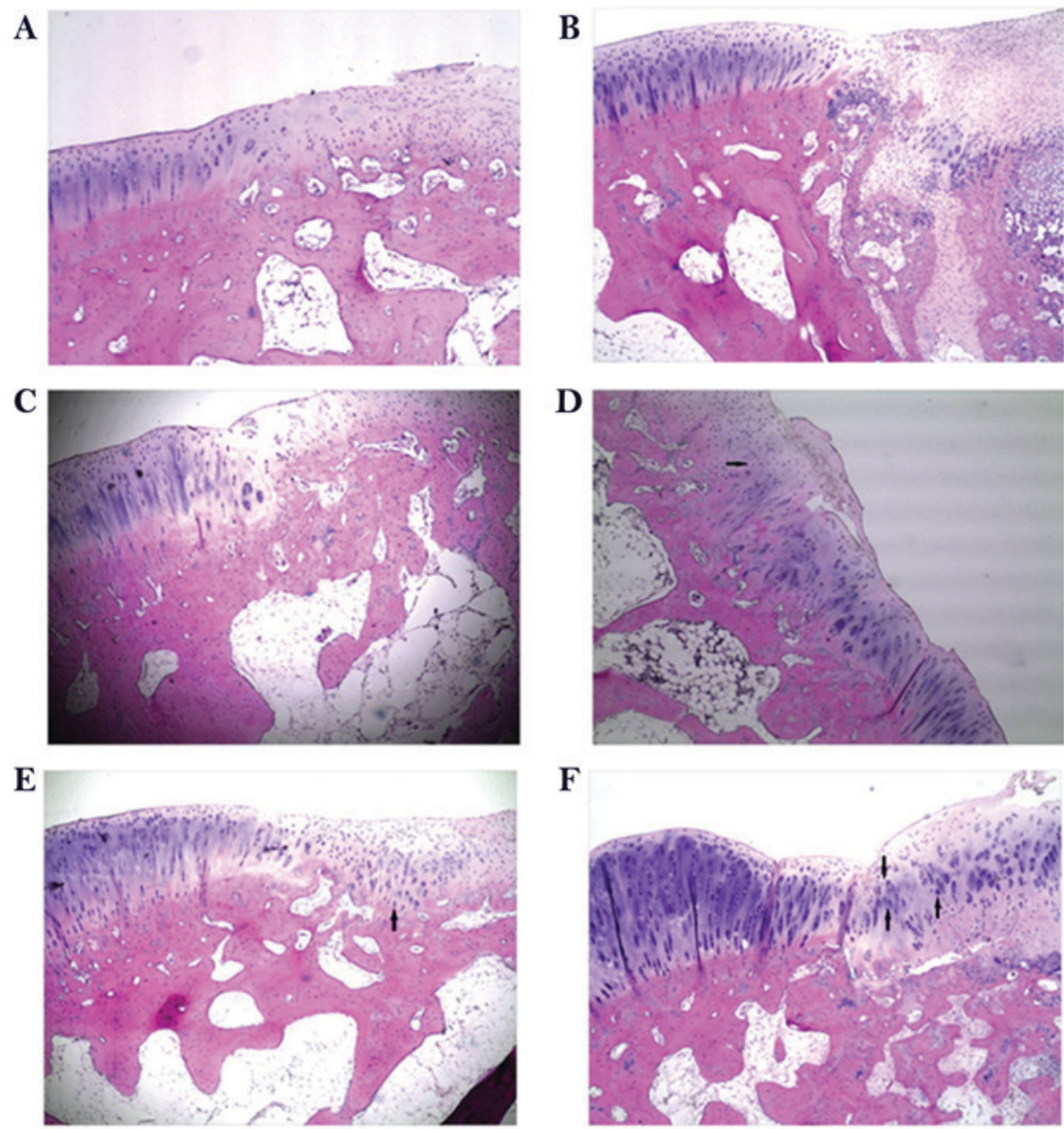

Figure 5. Hematoxylin-eosin staining following surgery. Representative images of the untreated control at (A) 6 weeks and (B) 12 weeks after surgery Representative images of the scaffold group at (C) 6 weeks and (D) 12 weeks after surgery. Representative images of the scaffold and radial extracorporeal shock wave therapy group at (E) 6 weeks and (F) 12 weeks after surgery (magnification, x40). Arrows indicate chondrocyte-like cells.

injury (22-25); however, the mechanisms underlying its effect remain largely unknown.

In the present study, rESWT was used to treat osteochondral defects. Promising results were obtained in the scaffold plus rESWT group, compared with the scaffold group, as demonstrated by the higher ICRS scores and improved gross appearance, which were much more evident at 12 weeks after surgery.

Nitric oxide (NO) is one of the main biological mediators under acoustic stimulation by shock waves (26). It has been confirmed that the systemic concentrations of $\mathrm{NO}$ increased at 1 month after ESWT was conducted for the treatment of on long bone nonunion (27). These results suggested the potential role of NO in ESWT. NO is important in OA progression and mediates the inflammatory responses. It is also involved in the apoptosis of chondrocytes, stimulation of matrix metalloproteinases, and degradation of collagen and proteoglycans (28-31). In the current study, NO levels in the synovial cavity were significantly reduced subsequent to treatment using a scaffold alone or scaffold plus rESWT at 6 weeks after surgery compared with the control group, while no significant difference was detected between the two groups. However, the mean value was lower when rESWT was used; since NO may serve a role in treatment with rESWT, further examination should be conducted in the future. Furthermore, the NO levels were not markedly different among the three groups at 12 weeks after surgery. This finding was not consistent with the observations of the study by Zhao et al (31), which mentioned that NO production was reduced in a rabbit OA model at 4 and 8 weeks after ESWT. The NO level in this previous OA model was markedly higher in comparison with that in the model of the present study. The discrepancy in these results may be due to the following reasons: Firstly, the OA model established in the previous study was induced by anterior cruciate ligament transaction, while the current study model was induced by drilling a hole in femoral condyle; secondly, the previous authors selected 4 and 8 weeks as the time points of NO detection, whereas 6 and 12 weeks were selected in the present study; thirdly, although rESWT was used in the two studies, different manufacturer, frequency and energy flux density may have caused different results; and finally, the use of a different method and kit may have also given rise to this discrepancy. NO is an important factor in osteochondral injury. It is routinely examined through quantification of the concentrations of nitrite/nitrate (NOX) using a method based on the enzymatic conversion of nitrate to nitrite facilitated by a nitrate reductase and followed by colorimetric detection of nitrite using a Griess reaction (32). As the detection procedure and incubation durations are not consistent between various laboratories, the concentrations of NOX will differ. Furthermore, several biomarkers have been reported to be involved in the pathogenesis of osteochondral injury or OA, such as prostaglandin E2 $(33,34)$, and the 
A

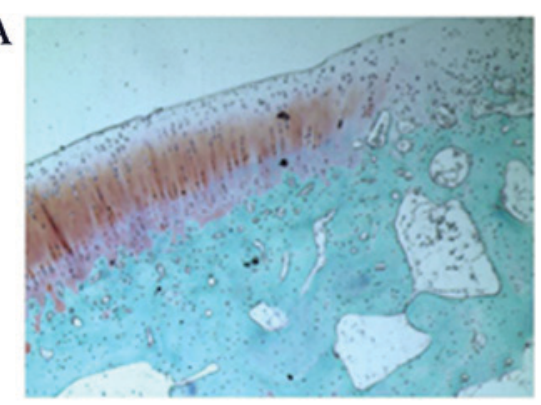

C

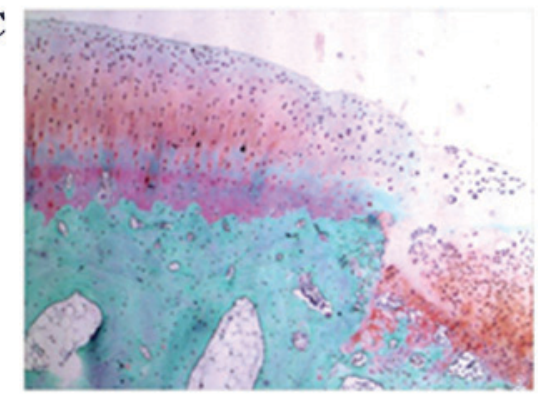

E

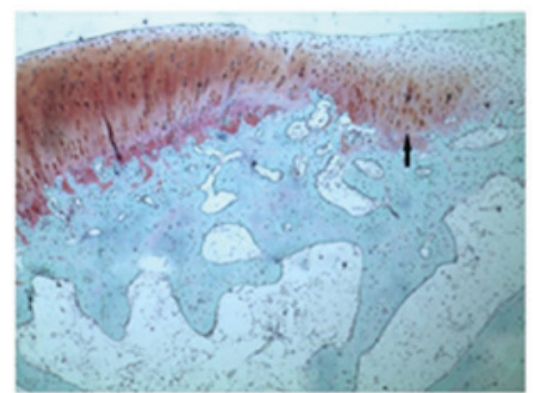

B

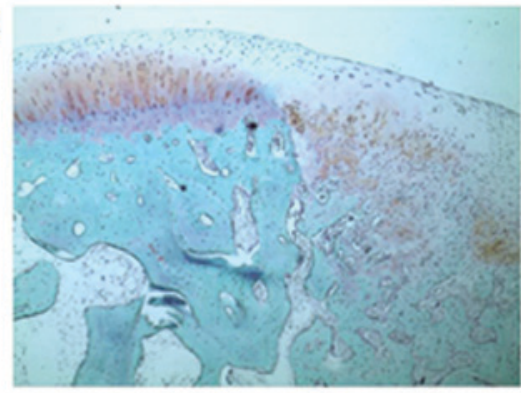

D

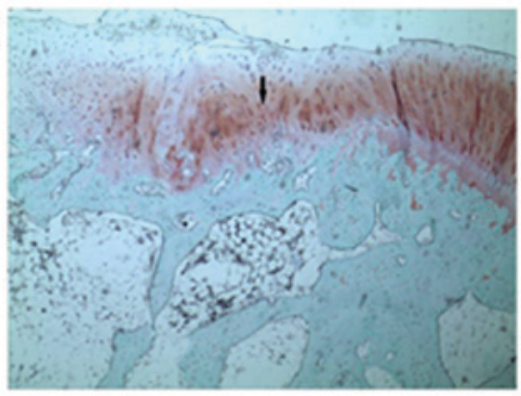

F

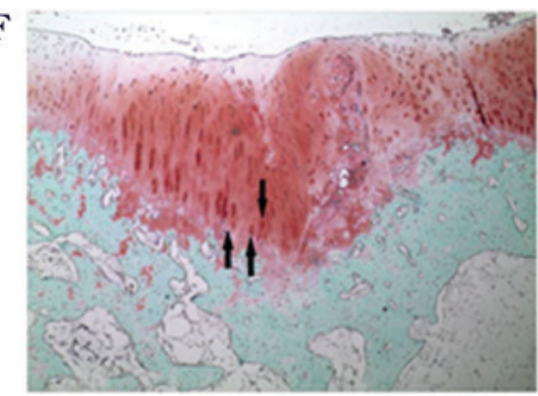

Figure 6. Safranin O/fast green staining following surgery. Representative images of the untreated control group at (A) 6 weeks and (B) 12 weeks after surgery. Representative images of the scaffold-implanted group at (C) 6 weeks and (D) 12 weeks after surgery. Representative images of the scaffold and radial extracorporeal shock wave therapy group at (E) 6 weeks and (F) 12 weeks after surgery (magnification, x40). Arrows indicate chondrocyte-like cells. Arrows indicate chondrocyte-like cells.

results of the present study will be further verified using such biomarkers in the future. To further clarify the underlying molecular mechanisms, other effects of rESWT on chondrocytes in vitro will also be assessed.

The current study observed that rESWT promoted osteochondral regeneration in rabbits, which may in part be due to the reduction of NO level in the synovial fluid in the knee joint; however, the exact molecular mechanisms remain unknown. In the present study, only a single dose was assessed and one treatment of rESWT was conducted; therefore, it is unclear whether other energy levels or multiple applications of rESWT have the same effect. The time point at which rESWT was conducted may also be an important factor, and certain researchers have suggested performing rESWT on knee OA prior to surgical treatment (25). Therefore, further in vitro and in vivo studies need to be conducted in the future, both basic and clinical, although the important role of rESWT on osteochondral regeneration is evident, and this strategy may serve as a valuable alternative supplement.

In conclusion, due to inadequate blood supply and limited regeneration ability of cartilage, there is a lack of effective treatments for osteochondral defects. Emerging studies have revealed the excellent therapeutic efficacy of ESWT on bone regeneration. Similarly, the results of the present study indicated that rESWT combined with the use of a scaffold was able to improve osteochondral regeneration. At 6 weeks after surgery, NO levels in the scaffold and the scaffold plus rESWT groups were significantly reduced in the synovial cavity, whereas no significant difference was detected at 12 weeks after surgery. Furthermore, the ICRS scores and histological examination also revealed a much improved osteochondral regeneration following treatment with scaffold plus rESWT. These data suggest that rESWT improved osteochondral regeneration when combined with a scaffold and that it may be a useful treatment for osteochondral defects.

\section{Acknowledgements}

Not applicable.

\section{Funding}

The present study was funded by the Capital Public Health Project (grant no. Z171100000417025) and the National Natural Science Foundation of China (grant no. 91543124).

\section{Availability of data and materials}

All data generated or analyzed during the present study are included in this article. 


\section{Authors' contributions}

YL, LS and HQ designed the project and supervised the study. HQ, SJ and LC performed the experiments. CY and YL analyzed the data and performed statistical analysis. HQ, SJ and CY generated the figures. HQ and YL wrote the manuscript. All authors read and approved the final version of the manuscript.

\section{Ethics approval and consent to participate}

The experimental protocol was reviewed and approved by the Institutional Review Board of Beijing Jishuitan Hospital (Beijing, China).

\section{Patient consent for publication}

Not applicable.

\section{Competing interests}

The authors declare that they have no competing interests.

\section{References}

1. Huey DJ, Hu JC and Athanasiou KA: Unlike bone, cartilage regeneration remains elusive. Science 338: 917-921, 2012.

2. Marcacci M, Filardo G and Kon E: Treatment of cartilage lesions: What works and why? Injury 44 (Suppl 1): S11-S15, 2013.

3. Filardo G, Andriolo L, Balboni F, Marcacci M and Kon E: Cartilage failures. Systematic literature review, critical survey analysis, and definition. Knee Surg Sports Traumatol Arthrosc 23: 3660-3669, 2015.

4. Okano T, Mera H, Itokazu M, Okabe T, Koike T, Nakamura H and Wakitani S: Systemic administration of granulocyte colony-stimulating factor for osteochondral defect repair in a rat experimental model. Cartilage 5: 107-113, 2014.

5. Khorshidi S and Karkhaneh A: A review on gradient hydrogel/fiber scaffolds for osteochondral regeneration. J Tissue Eng Regen Med 12: e1974-e1990, 2018

6. Kon E, Roffi A, Filardo G, Tesei G and Marcacci M: Scaffold-based cartilage treatments: With or without cells? A systematic review of preclinical and clinical evidence. Arthroscopy 31: 767-775, 2015.

7. Qi H, Jie YS, Chen L, Li QH, Gao XS and Sun L: Repair of articular defects in the knee with a cell-free scaffold. Orthopaedic Journal of China (Chinese) 23: 1303-1309, 2015.

8. Qi H, Jie YS, Chen L, Jiang J, Gao XS and Sun L: Preparation of acellular dermal matrix as a kind of scaffold for cartilage tissue engineering and its biocompatibility. Zhongguo Xiu Fu Chong Jian Wai Ke Za Zhi 28: 768-772, 2014 (In Chinese).

9. Qi H, Sun L, Chen L, Jie YS and Gao XS: Technics of hair removal-decellularization-reconstruction to build cartilage repair carrier. Orthopaedic J China (Chinese) 22: 151-157, 2014.

10. Qi H, Jie YS, Chen L, Tao JF, Jiang J and Sun L: Comparison of scaffolds for chondrocyte implantation with two kinds of cross-linking agents. Chin Med Biotechnol (Chinese) 8: 408-413, 2013.

11. Imamura M, Alamino S, Hsing WT, Alfieri FM, Schmitz $C$ and Battistella LR: Radial extracorporeal shock wave therapy for disabling pain due to severe primary knee osteoarthritis. J Rehabil Med 49: 54-62, 2017

12. Hochstrasser T, Frank HG and Schmitz C: Dose-dependent and cell type-specific cell death and proliferation following in vitro exposure to radial extracorporeal shock waves. Sci Rep 6: 30637, 2016.

13. Schmitz C, Császár NB, Milz S, Schieker M, Maffulli N, Rompe JD and Furia JP: Efficacy and safety of extracorporeal shock wave therapy for orthopedic conditions: A systematic review on studies listed in the PEDro database. Br Med Bull 116: 115-138, 2015.

14. Mainil-Varlet P, Aigner T, Brittberg M, Bullough P, Hollander A Hunziker E, Kandel R, Nehrer S, Pritzker K, Roberts S and Stauffer E: International Cartilage Repair Society. Histological assessment of cartilage repair: A report by the histology endpoint committee of the international cartilage repair society (ICRS). J Bone Joint Surg Am 85-A (Suppl)2: S45-S57, 2003.
15. Li X, Ding J, Wang J, Zhuang X and Chen X: Biomimetic biphasic scaffolds for osteochondral defect repair. Regen Biomater 2: 221-228, 2015.

16. Dahlin RL, Kinard LA, Lam J, Needham CJ, Lu S, Kasper FK and Mikos AG: Articular chondrocytes and mesenchymal stem cells seeded on biodegradable scaffolds for the repair of cartilage in a rat osteochondral defect model. Biomaterials 35: 7460-7469, 2014.

17. Jeon JE, Vaquette C, Theodoropoulos C, Klein TJ and Hutmacher DW: Multiphasic construct studied in an ectopic osteochondral defect model. J R Soc Interface 11: 20140184, 2014.

18. Armiento AR, Stoddart MJ, Alini M and Eglin D: Biomaterials for articular cartilage tissue engineering: Learning from biology. Acta Biomater 65: 1-20, 2018

19. Maia FR, Carvalho MR, Oliveira JM and Reis RL: Tissue engineering strategies for osteochondral repair. Adv Exp Med Biol 1059: 353-371, 2018.

20. Wang CJ: An overview of shock wave therapy in musculoskeletal disorders. Chang Gung Med J 26: 220-232, 2003.

21. Wang CJ: Extracorporeal shockwave therapy in musculoskeletal disorders. J Orthop Surg Res 7: 11, 2012.

22. Lyon R, Liu XC, Kubin M and Schwab J: Does extracorporeal shock wave therapy enhance healing of osteochondritis dissecans of the rabbit knee? a pilot study. Clin Orthop Relat Res 471: 1159-1165, 2013.

23. Frisbie DD, Kawcak CE and McIlwraith CW: Evaluation of the effect of extracorporeal shock wave treatment on experimentally induced osteoarthritis in middle carpal joints of horses. Am J Vet Res 70: 449-454, 2009.

24. Kim JH, Kim JY, Choi CM, Lee JK, Kee HS, Jung KI and Yoon SR: The dose-related effects of extracorporeal shock wave therapy for knee osteoarthritis. Ann Rehabil Med 39: 616-623, 2015.

25. Ochiai N, Ohtori S, Sasho T, Nakagawa K, Takahashi K, Takahashi N, Murata R, Takahashi K, Moriya H, Wada Y and Saisu T: Extracorporeal shock wave therapy improves motor dysfunction and pain originating from knee osteoarthritis in rats. Osteoarthritis Cartilage 15: 1093-1096, 2007.

26. Evens DM and Ralston SH: Nitric oxide and bone. J Bone Miner Res 11: 300-305, 1996.

27. Wang CJ, Yang KD, Ko JY, Huang CC, Huang HY and Wang FS: The effects of shockwave on bone healing and systemic concentrations of nitric oxide (NO), TGF-betal, VEGF and BMP-2 in long bone non-unions. Nitric Oxide 20: 298-303, 2009.

28. Abramson SB: Nitric oxide in inflammation and pain associated with osteoarthritis. Arthritis Res Ther 10 (Suppl 2): S2, 2008.

29. Hancock CM and Riegger-Krugh C: Modulation of pain in osteoarthritis: The role of nitric oxide. Clin J Pain 24: 353-365, 2008.

30. Studer R, Jaffurs D, Stefanovic-Racic M, Robbins PD and Evans CH: Nitric oxide in osteoarthritis. Osteoarthritis Cartilage 7: 377-379, 1999.

31. Zhao Z, Ji H, Jing R, Liu C, Wang M, Zhai L, Bai X and Xing G: Extracorporeal shock-wave therapy reduces progression of knee osteoarthritis in rabbits by reducing nitric oxide level and chondrocyte apoptosis. Arch Orthop Trauma Surg 132: 1547-1553, 2012.

32. Du Q, Park KS, Guo Z, He P, Nagashima M, Shao L, Sahai R, Geller DA and Hussain SP: Regulation of human nitric oxide synthase 2 expression by Wnt beta-catenin signaling. Cancer Res 66: 7024-7031, 2006.

33. Nguyen LT, Sharma AR, Chakraborty C, Saibaba B, Ahn ME and Lee SS: Review of prospects of biological fluid biomarkers in osteoarthritis. Int J Mol Sci 18: E601 2017.

34. Li N, Rivéra-Bermúdez MA, Zhang M, Tejada J, Glasson SS, Collins-Racie LA, Lavallie ER, Wang Y, Chang KC, Nagpal S, et al: LXR modulation blocks prostaglandin E2 production and matrix degradation in cartilage and alleviates pain in a rat osteoarthritis model. Proc Natl Acad Sci USA 107: 3734-3739, 2010.

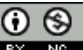

This work is licensed under a Creative Commons Attribution 4.0 International (CC BY-NC 4.0) License 